\title{
Vulnerabilidades de mulheres quilombolas no interior da Amazônia às infecções sexualmente transmissiveis: um relato de experiência
}

\author{
Vulnerabilities of maroon women inside the Amazon to \\ sexually transmitted infections: a report of experience
}

\author{
Veridiana Barreto do Nascimento',2, Nádia Vicência do Nascimento Martins ${ }^{1,3}$, \\ Suely Itsuko Ciosak', Lúcia Yasuko Izumi Nichiata', \\ Jéssica Samara dos Santos Oliveira ${ }^{4}$, Lays Oliveira Bezerra ${ }^{4}$, \\ Luana Almeida dos Santos ${ }^{5}$
}

'Programa de Pós-graduação em Enfermagem, Escola de Enfermagem, Universidade de São Paulo, São Paulo (SP), Brasil.

${ }^{2}$ Universidade Federal do Amapá, Oiapoque (AP), Brasil

${ }^{3}$ Universidade do Estado do Pará, Santarém (PA), Brasil.

${ }^{4}$ Curso de Enfermagem, Universidade do Estado do Pará, Campus XII, Santarém (PA), Brasil.

${ }^{5}$ Secretaria Municipal de Saúde de Santarém, Santarém (PA), Brasil.

Recebido: Ago. 14, 2017 Aceito: Nov. 09, 2017

\section{COMO CITAR ESTE ARTIGO} Nascimento VB, Martins NVN Ciosak SI et al. Vulnerabilidades de mulheres quilombolas no interior da Amazônia às infecções sexualmente transmissíveis: um relato de experiência. Interdisciplinary Journal of Health Education. 2017 Jan-Jul;2(1):68-73. http:// dx.doi.org/10.4322/ijhe.2016.029

\section{CORRESPONDÊNCIA}

Nádia Vicência do Nascimento Martins Travessa Professor Carvalho, 1420 Caranazal, CEP 68040-470, Santarém (PA), Brasil

stm.martins@hotmail.com

\section{FONTE DE FINANCIAMENTO \\ Realizada com financiamento}

próprio das pesquisadoras.

\section{CONFLITO DE INTERESSE}

Os autores declararam não

haver conflitos de interesse.

O estudo foi realizado nas Comunidades de remanescentes Quilombolas, localizadas no Alto Trombetas, município de Oriximiná (PA), Brasil.

Este trabalho é resultado do manuscrito "SABERES E VULNERABILIDADES DE MULHERES QUILOMBOLAS NO INTERIOR DA AMAZÔNIA SOBRE INFECCÕES SEXUALMENTE TRANSMISS UM RELATO DE EXPERIÊNCIA".

\section{RESUMO}

Contextualização: Relato de experiência derivado de uma prática vivenciada por docentes e discentes durante o período de coleta de dados de uma pesquisa de mestrado intitulada "Vulnerabilidades de mulheres quilombolas do rio Trombetas às IST/HIV/aids", no município de Oriximiná, Estado do Pará. Levando em consideração as necessidades em saúde as mulheres quilombolas, buscou-se como objetivo identificar as vulnerabilidades de mulheres quilombolas às Infecções Sexualmente Transmissíveis (IST). Descrição da experiência: Em outubro de 2016, uma equipe composta por docentes e discentes do curso de Enfermagem embarcou em uma viagem rumo às comunidades quilombolas, situadas nas encostas do Rio Trombetas. Durante as atividades de promoção de saúde desenvolvidas por meio de metodologias ativas de educação para 121 mulheres quilombolas, foram evidenciadas as fragilidades no conhecimento de mulheres quilombolas frente à prevenção das IST/HIV/aids, sendo potencializadas pela deficiência nos serviços e ações em saúde ofertados as comunidades. A experiência oportunizou um cenário de prática aos discentes do curso de Enfermagem, com uma aprendizagem voltada à realidade e humanização do cuidado, o que permitiu a troca de conhecimentos e orientações acerca de IST. Portanto, se pode inferir que, as mulheres quilombolas estão expostas nas três dimensões de vulnerabilidade: individual, social e programática quando se refere as IST/HIV/aids. Considerações finais: Apresentar ao acadêmico de Enfermagem a realidade de populações tradicionais amazônicas, possibilitou o conhecimento da situação de saúde e educação de populações tradicionais da Amazônia, levando ao crescimento pessoal e acadêmico, aguçando no futuro profissional, uma visão crítica e reflexiva para mudança do cenário atual da Saúde Coletiva Brasileira, favorecendo a efetivação dos serviços e ações em saúde nas comunidades por meio de Políticas Públicas.

PALAVRAS-CHAVE: Atenção básica. Enfermagem. Infecções sexualmente transmissíveis. Saúde da mulher. População negra.

\section{ABSTRACT}

Contextualization: Report of experience derived from a practice experienced by professors and students during the data collection period of a master course study entitled "Vulnerabilities of Maroon women from the Coast of Trombetas River (PA) to STI/HIV/AIDS", in the municipality of Oriximiná, State of Pará. Taking into account the health needs to which maroon women are exposed, the objective was to identify the vulnerabilities of Maroon women to Sexually Transmitted Infections (STI). Description of the experience: In October 2016, a team of professors and students of the Nursing course embarked on a trip towards the maroon communities, located on the coast of the Trombetas River. During the health promotion activities, developed through active education methodologies for 121 aroon women, the weaknesses in the knowledge of maroon women against STI/HIV/AIDS prevention were evidenced, empowered by the deficiency in the health services and actions offered to the communities, in addition to providing a scenario of practice to the students of the Nursing course, with a learning focus on reality-oriented and humanized of care, which allows the exchange of knowledge and guidance about STI. Therefore, it can be inferred 
that maroon women are exposed in the three dimensions of vulnerability: individual, social and programmatic when referring to STI/HIV/AIDS. Final considerations: In order to show the reality of traditional Amazon populations to the Nursing student makes possible the knowledge of the health situation and their education, leading to personal and academic growth, fostering in the future professional a critical and reflexive vision to change the current scenario of Brazilian Collective Health. This type of actions favors the implementation of services and actions in the health of the communities through Public Policies.

KEYWORDS: Basic attention. Nursing. Sexually transmitted infections. Women's health. Black population.

\section{Contextualização}

Quilombo é um termo que remonta o período da escravidão, uma forma de resistência cultural e de rebeldia contra os valores impostos pelos brancos. Significa ter liberdade à vida, para cultuar sua religião, realizar suas danças (oriundas da África), iniciar plantações em terras livres e não estarem subjugados aos senhores de engenho ${ }^{1}$.

Os primeiros quilombos eram qualificados como um "ajuntamento" de negros fugidos que adentravam a mata, procurando territórios isolados e resistentes à captura, as populações sobreviventes deste processo originaram as atuais comunidades remanescentes de quilombo ${ }^{1}$.

As comunidades remanescentes de quilombos foram reconhecidas em 1988 na Constituição Federal Brasileira considerando, sobretudo, o direito ao território adquirido $^{2}$.

No âmbito da saúde, a luta começou em 1996 com um evento realizado em Brasília intitulada "Saúde da População Negra", onde começou os primeiros trabalhos em prol da saúde da população quilombola. No entanto, somente em 2004 foi instituída a Portaria $\mathrm{n}^{\circ} 1.434$ que objetiva a inserção e a ampliação das Equipes de Estratégias de Saúde para as comunidades quilombolas, garantido o princípio de Universalidade do Sistema Único de Saúde².

Os avanços nos serviços de saúde tornaram-se visíveis, somente a partir da Política Nacional de Saúde Integral da População Negra, instituída pela portaria No 992 de 2009, entretanto, um dos desafios na implementação das ações junto a estas comunidades é o acesso a informação de forma clara, objetiva e oportuna sobre saúde e, em particular sobre as Infecções Sexualmente Transmissíveis (IST) ${ }^{3}$.

Historicamente, mulheres brasileiras enfrentam obstáculos em virtude do preconceito e dos processos vinculados à dinâmica de gênero e não é diferente, se as colocarmos no contexto das relações sexuais e reprodutivas. Questões sociais tais como multiplicidade de parceiros sexuais, assimetria nas relações entre mulheres e homens, dogmas religiosos e implicações morais, expõe o público feminino às IST, concomitantemente, acentua a vulnerabilidade das mulheres. As mulheres negras remanescentes quilombolas ainda vivenciam esta assimetria de gênero, quando ligadas ao passado de escravidão $0^{4,5}$.

No Estado do Pará, a maioria das comunidades remanescentes de quilombos, encontram-se as margens do Rio Trombetas, no município de Oriximiná. Os negros que habitavam essas comunidades fugiam dos cocais, das fazendas e das propriedades dos senhores de Óbidos, Santarém, Alenquer e Belém durante o período das "cheias dos rios", assim como durante o período da colheita da Castanha-do-Pará, que lhes servia como alimento durante esta atividade. Ao encontrarem o Alto Trombetas, era possível ser livre, viver sua cultura e usufruir de uma vida tranquila ${ }^{1}$. É na cidade de Oriximiná que na atualidade se concentra grande parte dos remanescentes quilombolas deste estado.

Neste estudo, apresentam-se dados parciais de uma pesquisa de mestrado acadêmico, que tem por objetivo identificar a vulnerabilidade de mulheres quilombolas a Infecções Sexualmente Transmissíveis (IST). 


\section{Descrição da experiência}

Trata-se de um estudo descritivo, do tipo relato de experiência, com abordagem quantitativa, realizado com 121 mulheres quilombolas, residentes em 08 (oito) comunidades do Alto Trombetas, Pará: Serrinha, Terra Preta, Aracuan de Baixo, Aracuan do meio, Aracuan de cima, Bacabal, Boa Vista e Água Fria. As localidades detêm uma situação socioeconômica precária e para maioria da população residente, a fonte de renda está relacionada à agricultura de subsistência e a pesca.

Foram critérios de inclusão: mulheres autodeclaradas quilombolas, orientadas no tempo e no espaço no momento de participar das etapas da pesquisa (aplicação de questionário e entrevista); com vida sexual ativa e na faixa etária de 15 a 55 anos. E critérios de exclusão: mulheres com idade inferior a 15 anos e superior a 55 anos, na faixa etária de 15 a 18 anos não autorizada pelos pais ou responsáveis para participar da pesquisa e, as mulheres ausentes das comunidades no período da coleta de dados.

Para a coleta de dados foram utilizados, um questionário e um roteiro de perguntas para entrevista, elaborados para este estudo, composto por perguntas com respostas fechadas e abertas. Os dados foram organizados em planilhas no programa Microsoft Excel 2013 e para análise das variáveis utilizou-se o programa SPSS, versão 21.0.

As atividades foram desenvolvidas em três etapas no período de 9 a 15 de outubro de 2016, por uma equipe composta por docentes e acadêmicos do Curso de Enfermagem da Universidade do Estado do Pará - Campus XII Santarém, a primeira etapa iniciou com o agendamento de uma reunião com os representantes das comunidades para a apresentação da equipe de pesquisadores que, por sua vez apresentou o projeto de pesquisa para os comunitários, seus objetivos, os riscos e benefícios. Na segunda etapa, foi realizada a aplicação do questionário e a entrevista com objetivo de identificar contextos de vulnerabilidades de mulheres quilombolas às IST/HIV/aids. Na terceira etapa foram desenvolvidas atividades educativas nas comunidades, utilizando metodologias ativas de aprendizagem com temas voltados para a prevenção das IST.

Simultaneamente a aplicação do questionário de pesquisa, realizado por meio de visitas domiciliarias durante o dia, aconteceram às atividades de educação em saúde no período noturno, realizadas por discentes da UEPA, as atividades educativas desenvolvidas ocorreram a partir do seguinte roteiro: foram disponibilizadas imagens das IST dentro de balões (optou-se por imagens ao invés de perguntas ou afirmações, por considerar mais didática e facilitar a participação de um maior número de pessoas, incluindo as mulheres não alfabetizadas). As mulheres deveriam identificar os sinais, sintomas, modos de prevenção e transmissão das IST. Para o aprofundamento da temática, por meio de orientações automaticamente os conhecimentos eram intensificados com a apresentação de um painel identificando os sinais e sintomas das diversas IST, a forma de transmissão "como se pega e como não se pega", a prevenção e os mitos envolvidos.

Portanto, quando associado às repostas dos questionários e as entrevistas, aliado a participação das mulheres nas atividades de educação em saúde, os conhecimentos puderam ser construídos de forma simples, direta e esclarecedora, considerando os conhecimentos prévios de cada uma e facilitando inclusive a ação dos mediadores, que encontraram pouca dificuldade na abordagem do tema.

Alguns desafios acompanharam a evolução das atividades, principalmente as condições de acomodação para os pesquisadores, que se restringia a um barco de pequeno porte, porém as dificuldades vivenciadas não impediram a progressão das atividades, somado a experiência positiva para toda a equipe de execução do projeto, possibilitando a aproximação com complexidade no contexto ético racial, social, cultural e histórico, entrelaçada ao processo saúde e doença da população loco regional. 
O projeto de pesquisa que embasou este relato de experiência foi avaliado e aprovado pelo Comitê de Ética em Pesquisa da Escola de Enfermagem da Universidade de São Paulo, sob parecer de número 1.667.309.

Participaram da pesquisa 121 mulheres, residentes em 08 comunidades destas, a que teve menor número de participantes foi representada por 07 mulheres e a que teve maior número de participantes foi representada por 44 mulheres, a idade média das mulheres participante foi de 30 anos, autodeclaras negras e pardas em sua maioria, estado civil casada e união estável e com a conclusão máxima do ensino fundamental. A maioria das relações maritais aconteceu em idade precoce, antes de atingir a maioridade (18 anos).

Referente à religião as mulheres quilombolas em sua maioria declararam-se católicas. No que tange a moradia $44,6 \%$ residiam em casa de alvenaria com menos de três cômodos, com água para consumo proveniente do rio ou de microssistema comunitário. Apenas 38,1\% das mulheres quilombolas referiram realizar atividades remuneradas, declarando uma renda mensal individual menor que um salário mínimo vigente (ano 2016).

Sobre o conhecimento das IST, as respostas mostram que as mulheres conhecem as formas de prevenção e reconhecem o preservativo como um método eficaz e efetivo para a prevenção das IST/HIV/aids. A maioria declarou ter tido algum tipo de informação sobre IST, sendo a escola, o local de maior concentração de divulgação de conteúdos educativos sobre o tema, seguido das igrejas locais e associações. Quanto às formas de transmissão das IST indicaram a relação sexual sem preservativo, seguido do uso de seringas contaminadas e de contaminação por meio do contato com secreção vaginal e/ou secreção uretral.

A maioria das mulheres quilombolas tem percepção sobre o próprio risco de contrair IST/HIV/aids e que este risco é acentuado quando há infidelidade do parceiro. Outras têm a percepção de risco zero, ao afirmarem que "transariam sem camisinha".

\section{Resultados e impactos}

A partir dos resultados obtidos e descritos a seguir, fica evidenciado que as mulheres quilombolas participantes deste estudo estão inseridas nas três dimensões de vulnerabilidade (individual, social e programática), de acordo com Ayres et al. ${ }^{6}$ no eixo ligado a prevenção das IST/HIV/aids.

Nas comunidades quilombolas é comum mulheres jovens se relacionar com homens mais velhos, o que aumenta cerca de 10 vezes as chances de desenvolver IST/aids, evidenciando maior vulnerabilidade dessas mulheres devido à baixa escolaridade, trata-se de uma triste realidade, porém, muito comum nas comunidades da Amazônia Brasileira. Nestas comunidades o ensino funciona em formato modular e as escolas não disponibilizam o ensino médio, logo se pode inferir que o problema está relacionado ao acesso aos serviços educacionais ${ }^{7}$.

A baixa escolaridade, as desigualdades de gênero, o baixo nível socioeconômico e as disparidades regionais podem ser considerados fatores condicionantes para a vulnerabilidade individual feminina as IST/HIV/aids, na qual o conhecimento sobre as formas de transmissão e prevenção dessas infecções é incipiente e aumenta o risco de infecção $0^{8,9}$.

Este fato chama atenção, haja vista que mesmo as pesquisandas detendo conhecimento de que o ato sexual sem proteção aumenta as chances de transmissão das IST, as mulheres, em sua maioria, não utilizam o preservativo. Neste contexto, nota-se que apesar das mulheres conhecerem informações sobre IST/HIV/aids, o uso do preservativo ainda é baixo entre a população sexualmente ativa, independentemente do seu estado civil.

Assim, se enfatiza que a carência de informações a respeito das IST são fatores que favorecem as concepções errôneas e influenciam diretamente o comportamento feminino. Tais equívocos conceituais podem ser fundamentados em elementos 
culturais como, por exemplo, crenças, mitos e tabus que apresentam grande significado para os indivíduos, não representando fontes corretas e confiáveis de informações ${ }^{10,11}$.

O conhecimento adequado sobre às IST/aids, em especial sobre sua forma de transmissão e prevenção, auxilia na redução da vulnerabilidade na exposição ao vírus HIV. Vale ressaltar que tanto no Brasil quanto na América Latina, a epidemia da aids apresenta maiores índices entre a população feminina, mais jovem e pobre. A síndrome afeta cada vez mais mulheres que adquirem o vírus em suas próprias casas através dos maridos ou companheiros ${ }^{12,13}$.

Sabe-se que a ausência ou mesmo limitação de acesso aos de serviços de saúde e educação na comunidade retratam desigualdades e vulnerabilidades na sociedade, em especial desta população, que vivencia um contexto diferente de escala geográfica, enfrentando grandes distâncias para acessar os serviços de saúde e educação indispensáveis a população ${ }^{14}$.

Na região estudada, as condições de saneamento básico, moradia, acesso aos serviços de saúde, educação e ao trabalho local são deficitárias, o que maximiza as fragilidades e limitações da população quilombola e evidencia as inúmeras peculiaridades no que tange o acesso à saúde desta população, levando a necessidade de maior planejamento e implementação de estratégias sejam elas coletivas e ou individuais concernentes às particularidades quilombolas.

\section{Considerações finais}

A deficiência ou mesmo inexistência de uma equipe de profissionais de saúde, a dificuldade de deslocamento, seja para o polo de assistência em saúde na cidade de Oriximiná ou para o hospital da mineração são atenuantes expressivos. Em algumas comunidades os moradores podem contar com a presença de Agentes Comunitários de Saúde (ACS), que são acionados em uma situação emergencial e nas práticas de orientação em saúde e na identificação de agravos. Nesta pesquisa, a atuação dos ACS foi de grande importância, pois, facilitaram a ação dos pesquisadores, fornecendo dados sobre o perfil da comunidade, realizando a mobilização das mulheres por meio de convite e estímulo para a participação nas atividades. Na comunidade quilombola o ACS é visto e, funciona como ponte/elo de conhecimento em saúde e primordialmente na prevenção de agravos.

Neste contexto desafiador conclui-se que os objetivos propostos foram alcançados. O emponderamento das mulheres sobre o conhecimento das IST/HIV/aids por meio de educação em saúde de forma inovadora, dinâmica e reflexiva, permitiu a troca de experiências acerca das IST, despertando o interesse da população em explorar cada vez mais conhecimentos acerca do assunto, além de permitir maior desenvoltura aos alunos de graduação, possibilitando, ainda, crescimento pessoal e profissional para o enfrentamento e tomada de decisão enquanto profissionais de saúde nas diversas realidades brasileiras. Realidades estas, que guardam consigo parte de um arcabouço cultural, ético racial e social, tão importante na formação do Brasil e que necessita urgentemente de valorização e cuidados específicos, pelas autoridades, principalmente da área de saúde.

\section{Referências}

1. Funes EA. Mocambos: natureza, cultura e memória. História Unisinos. 2009;13(2):146-53. http://dx.doi.org/10.4013/ htu.2009.132.05.

2. Fundação Cultural Palmares [Internet]. Brasília; 2015 [citado em 2015 Set 27]. Disponível em: http://www.palmares.gov. br/?page_id $=88$

3. Brasil. Ministério da Saúde. Secretaria de Vigilância em Saúde. Departamento de DST, Aids e Hepatites Virais. Boletim epidemiológico HIV/Aids [Internet]. 2015 [2017 Maio 10];4(1). Disponível em: http://www.aids.gov.br/en/node/57749 


\section{ijhe}

4. Silva MHP. Assistência à saúde em comunidades quilombolas: revisão sistemática [monografia]. Salvador: Faculdade de Medicina, Universidade Federal da Bahia; 2015 [citado em 2017 Jun 1]. Disponível em: https://repositorio.ufba.br/ri/ bitstream/ri/18346/1/ Marcos\%20Henrique\%20Paraiso\%20Silva.pdf

5. Freitas DA, Caballero AD, Marques AS, Hernández CIV, Antunes SLNO. Saúde e Comunidades Quilombolas: uma revisão da literatura. Rev CEFAC. 2011;13(5):937-43. http://dx.doi.org/10.1590/S1516-18462011005000033.

6. Ayres JRCM, França I Jr, Calazans GJ, Saletti FHC. O conceito de vulnerabilidade e as práticas de saúde: novas perspectivas e desafios. In: Czeresnia D, Freitas CM, organizadores. Promoção da saúde: conceitos, reflexões, tendências. Rio de Janeiro: Fiocruz; 2003. p. 117-39.

7. Braga PE, Cardoso MRA, Segurado AC. Diferenças de gênero ao acolhimento de pessoas vivendo com HIV em serviço universitário de referência de São Paulo, Brasil. Cad Saude Publica. 2007;23(11):2653-62. http://dx.doi.org/10.1590/S0102311X2007001100013. PMid:17952258.

8. Arruti JMA, Monteiro JM. Mocambo: antropologia e história do processo de formação quilombola. Bauru: Edusc; 2006.

9. Miranda-Ribeiro P, Simão AB, Caetano AJ, Lacerda MA, Torres MEA. Perfis de Vulnerabilidade Feminina ao HIV/aids em Belo Horizonte e Recife: comparando brancas e negras. Saude Soc. 2010;19(Supl. 2):21-35. http://dx.doi.org/10.1590/ S0104-12902010000600004.

10. Panobianco MS, Lima ADF, Oliveira ISB, Gozzo TO. O conhecimento sobre o HPV entre adolescentes estudantes de graduação em enfermagem. Texto Contexto Enferm. 2013;22(1):201-7. http://dx.doi.org/10.1590/S0104-07072013000100024.

11. Santos MF. Concepções de mitos e relações com a saúde. Bol Mus Int Roraima. 2014;8(2):53-60.

12. Scherer A, Coelho EBS, Santos CFT. Conhecimento e Atitudes de Mulheres sobre AIDS: uma difícil negociação. Saúde Transform Soc. 2011;1(2):33-42.

13. Dornelas J No, Santos DAC, Zonta GE, Bonafé SM. Situação do hiv/aids no brasil e os fatores que influenciam a infecção. In: Anais Eletrônico do VIII EPCC - Encontro Internacional de Produção Científica [Internet]; 2013 out 22-25; Maringá. Maringá (PR): UNICESUMAR; 2013 [2017 maio 27]. Disponível em: http://www.cesumar.br/prppge/pesquisa/epcc2013/ oit_mostra/Jader_Dornelas_Neto.pdf

14. Stopa SR, Malta DC, Monteiro CN, Szwarcwald CL, Goldbaum M, Galvão Cesar CL. Acesso e uso de serviços de saúde pela população brasileira, Pesquisa Nacional de Saúde 2013. Rev Saude Publica. 2017;51(Supl. 1):1s-11s.

\section{Contribuição dos autores}

Todos os autores participaram do planejamento e execução do trabalho. 